\title{
Organic Ion Imaging Beyond the Limit of Static Secondary Ion Mass Spectrometry
}

\author{
J. M. McMahon, N. N. Dookeran, and P. J. Todd \\ Chemical and Analytical Sciences Division, Oak Ridge National Laboratory, Oak Ridge, Tennessee, USA
}

\begin{abstract}
Secondary ion mass spectra and images were obtained from spikes of choline chloride, acetylcholine chloride, and methylphenylpyridinium iodide deposited onto specimens of porcine brain tissue. Samples were subsequently subjected to a dose of $10-\mathrm{keV} \mathrm{Cs}^{+}$sufficient to suppress secondary ion emission characteristic of the targeted analytes. Following ablation of the samples by massive glycerol clusters generated by electrohydrodynamic emission, secondary ion mass spectra and images could be obtained that reflected the identity and location of the spiked analytes. The absolute intensity of secondary ion emission that followed ablation was found to be between 30 and $100 \%$; of the intensity obtained prior to exposure to the high dose of $\mathrm{Cs}^{\prime}$. Not all chemical noise is removed by ablation, however, so that the signal-to-noise ratios after ablation correspond to between 10 and $85 \%$ of their values observed under conditions of low primary ion dose. (f Am Soc Mass Spectrom 1995, 6, $1047-10581$
\end{abstract}

$\mathrm{I}$

n 1978, Benninghoven [1] reported that under conditions of limited exposure to a beam of kiloelectronvolt primary ions, involatile organic compounds emitted secondary ions characteristic of their structure. Since then, one of the goals of research in secondary ion mass spectrometry (SIMS) has been to apply the imaging capabilities of SIMS to map the distributions of targeted compounds in a variety of matrices, particularly biologic tissue. The virtual explosion of other biomedical imaging methods, such as autoradiography, positron emission tomography, magnetic resonance imaging, and so forth, over the past decade attests to the biomedical utility of mapping biologic tissue. There is compelling scientific and economic motivation for research to identify and map the distribution of drugs, toxins, and their metabolites in biologic tissue with greater spatial resolution, specificity, and sensitivity. Because it is a mass spectral method, SIMS offers unique specificity and sensitivity compared to other biomedical imaging methods, particularly when used in combination with tandem mass spectrometry [2].

Despite the motivation and potential, progress toward the application of SIMS to biomedical imaging has been hampered by a variety of barriers. Among the most important of these barriers has been the so-called static SIMS limit. Secondary ion imaging depends upon the impact of a highly focused and rastered primary ion beam on the sample. Although there can be large variation among different types of samples, organic

Address reprint requests and correspondence to Peter J. Todd, Oak Ridge National Laboratory, P.O. Box 20108, Bldg. 5510A, Oak Ridge, TN 37831-6.365. samples cease to emit characteristic secondary ions after they have been exposed to a dose of about $10^{13}-10^{14}$ primary ions $/ \mathrm{cm}^{2}$ [3]. Generally, static SIMS spectra are obtained by using a dose of primary ions that is $10 \%$ of this value. This dose corresponds to analysis of only a few percent of the molecules located on the surface of an organic sample. The static SIMS limit not only restricts the sensitivity of the method, but also precludes use of tandem mass spectrometry and depth profiling. Depth profiling is widely used with inorganic samples to produce three-dimensional maps. Secondary ion microprobes can only detect ions from a small area of the sample surface, typically $<1 \mathrm{~mm}^{2}$. To put the effect of the static SIMS limit into perspective, the largest sample that can be analyzed by static SIMS microprobe is approximately 1 pmol.

There are, of course, organic compounds that apparently are immune to the ravages of the primary ion beam. These compounds include a number of involatile liquids such as glycerol and the other compounds used as solvents (or matrices) to dissolve compounds prior to ionization by fast-atom bombardment (FAB) [4]. With such solutions, characteristic secondary ion emission persists well beyond the static SIMS limit, until the liquid evaporates. Very early in the investigation of secondary ion emission from liquids, Röllgen and co-workers [5] pointed out that liquids such as glycerol must facilitate some kind of surface "selfcleaning" mechanism to remove the products of primary ion damage. We employed imaging SIMS to show that the reason these liquids are apparently immune to primary ion damage is that a Marangoni effect, that is, surface tension-driven mass transport, drives the detritus of primary ion damage to the pe- 
riphery of the droplet, leaving a "clean" liquid surface exposed to the primary ion beam [6]. Such a mechanism was proposed by Ligon and Dorn [7] based upon the FAB mass spectra of surfactant solutions.

Clearly, one way to circumvent the problem of primary ion damage and the static SIMS limit is to remove the products of the reactions induced by the primary ion beam. The trick is to perform this operation without destruction of the spatial integrity of the samples. By using low energy primary ions, secondary ion emission characteristic of organic samples deposited on metal apparently can be generated indefinitely, and it has been suggested that more massive primary ions could permit depth profiles [8]. Recently, Cornet et al. [9] reported that by exposing dry samples of involatile organic compounds to a beam of massive glycerol cluster ions and neutrals, mass spectra reflective of the sample could be obtained continuously for periods of up to $1 \mathrm{~h}$, presumably well beyond the static SIMS limit. The beam of clusters is formed by electrohydrodynamic emission (EHD) from a solution of glycerol and ammonium acetate [10]. Reportedly the clusters possess a range of molecular weights that extend to $10^{7} \mathrm{u}$ and a range of kinetic energy that extends to $10^{6} \mathrm{eV}$. Upon striking the sample, a large amount of energy is deposited within a region near the surface and within the proximate area of each cluster [11]. This deposition permits generation of multiply charged secondary ions with molecular weights in excess of $10^{4}$. Despite these large numbers, the energy per nucleon corresponds to about $1 \mathrm{eV} / \mathrm{u}$ or $12 \mathrm{eV}$ for carbon atoms, 16 for oxygen atoms, and so forth. For such kinetic energy, the stopping power of organic targets limits penetration (i.e., the range) of such projectiles to about $10 \AA$ or less. Thus, any kinetic energy transferred directly to a surface by the clusters is confined to the first $10 \AA$ of the surface. For comparison purposes, $10-\mathrm{keV} \mathrm{Cs}^{+}$that impacts on an organic surface is stopped by both electronic and nuclear stopping power of the organic target; only a small portion of its kinetic energy is deposited near the surface and penetration is typically to a depth on the order of $200 \AA$ [12]. Along its track, electronic and nuclear (i.e., displacement of atoms from target molecules) excitation damages the sample. Below the surface, damaged molecules necessarily are trapped and may react, which causes chemical damage.

Arguably, massive cluster ionization (MCI) produces persistent emission of secondary ions characteristic of the sample because the primary particles do not significantly penetrate the surface, and a large part of whatever damage is caused by the primary clusters evaporates. We have demonstrated (Dookeran, N. N.; McMahon, J. M.; Short, R. T.; Todd, P. J., unpublished) the capacity of massive cluster impact to "clean" samples of stearic acid prior to analysis by static SIMS. That is, after exposure to a beam of clusters, subsequent $\mathrm{Cs}^{+}$SIMS spectra and images from stearic acid show a reduction in chemical noise. Beams of massive clusters produced by $\mathrm{MCl}$ have a large kinetic energy distribution, a large distribution of charge states, and a significant component of high energy neutral clusters. Because of these features, such beams can neither be focused nor rastered to produce an ion micrograph. The surface cleaning capability of high energy massive clusters, however, might be useful to remove layers of an organic sample damaged by primary $\mathrm{Cs}^{+}$impact. That is, a massive cluster beam could be used in conjunction with a focused beam to obtain images beyond the static SIMS limit.

Cleaning the surface of biologic tissue damaged by a primary $\mathrm{Cs}^{+}$beam is a very different experiment than acquisition of $\mathrm{MCI}$ spectra over a prolonged period or cleaning adsorbed material from a neat organic surface. We have found [13] that the degradation of secondary ion emission from biologic tissue with primary ion dose often is more rapid than from neat organic samples. Either the primary $\mathrm{Cs}^{+}$ion beam may damage the sample beyond repair or the cluster beam itself may cause such damage that its beneficial effects are negligible. Second, damage caused by a $10-\mathrm{keV} \mathrm{Cs}{ }^{+}$primary ion beam extends more deeply into the sample than does any surface contamination or any damage caused by cluster impact. Thus, it is necessary that the cluster beam remove multiple layers between imaging by the primary $\mathrm{Cs}^{+}$beam.

The potential applications of the cluster beam led us to test its ability to rejuvenate secondary ion emission from biological tissue samples exposed to a dose of primary $\mathrm{Cs}^{*}$ in excess of the static SIMS limit. The behavior of secondary ion mass spectra and images as a function of primary $\mathrm{Cs}^{+}$dose was reported earlier for similar samples [13]. Generally speaking, both the total secondary ion current and the secondary ion current of ions characteristic of the analyte are dramatically reduced when the primary dose exceeds the static SIMS limit. The use of methods similar to those here, allowed us to show that such secondary ion emission can be rejuvenated by ablation of the sample with a beam of massive clusters.

\section{Experimental}

\section{Secondary Ion Mass Spectrometer}

The triple quadrupole mass spectrometer-microprobe has been described in detail elsewhere [14]. The primary ion column consists of a thermionic $\mathrm{Cs}^{+}$primary ion gun and an objective lens with raster capability. The primary beam can be focused to a spot size of about $100-\mu \mathrm{m}$ diameter, with a raster area of about 1.2 $\mathrm{cm} \times 1.2 \mathrm{~cm}$. The primary ion beam current and current density are measured by imaging the shadow of a high transmission grid placed over a Faraday cup, which for primary ion current measurement occupies the position normally occupied by a sample.

The massive cluster ion gun (Phrasor Scientific, Duarte, CA) consists of an EHD emitter electrode sys- 
tem (emitter and counterelectrode), a three element Einzel lens, a deflector system, and a power supply. The source, which is attached to a 2.75 high vacuum flange, was mounted a distance of $23 \mathrm{~cm}$ from the target center, such that the beam would strike the sample at an angle of $45^{\circ}$ with respect to the target normal. The massive cluster ion beam was generated by EHD from a solution of 1.5-M ammonium acetate in glycerol at an emitter potential of $15 \mathrm{kV}$. To initiate the flow of glycerol solution, the reservoir was heated slightly and the high voltage power supply was energized. The onset of emission is marked by an increase of about $1-2 \times 10^{-5}$ torr in the pressure of the microprobe vacuum housing, as indicated by an (uncalibrated) Bayard-Alpert gauge. After an initial period of erratic emission, typically lasting $10 \mathrm{~min}$, the emission current stabilized and the counterelectrode voltage was adjusted to bring the emission current to a nominal value of $10 \mu \mathrm{A}$. Under these conditions, the current of the beam, measured with a shielded Faraday cup, ( $1 \mathrm{~cm}$ diam) situated in the position normally occupied by the sample, showed a current of between 5 and $50 \mathrm{nA}$, dependent on focusing conditions. The distribution of the clusters that strike a sample was determined by coating a target with $\mathrm{ZnS}$ phosphor and loading it as if it were a sample. Interestingly, focusing sufficient to cause the tenfold change in measured current appears to have a minimal effect on the appearance of light emitted from the phosphor. In fact, the brightness of the $2-\mathrm{cm}$ spot emitted by the massive cluster beam is comparable to the brightness emitted by the same phosphor when struck by a $10-\mathrm{keV} \mathrm{Cs}{ }^{+}$ $10-\mu \mathrm{A} / \mathrm{cm}^{2}$ beam. These results seem to suggest that a significant portion of the cluster beam is, in fact, neutrals.

The cluster beam was aimed at the target by first loading a sample of choline chloride onto a target, setting the quadrupole mass filters to transmit only the $m / z 104$ secondary ions, and setting the dynamic emittance matching of the secondary ion source to transmit ions only from the center of the target. By alternate adjustment of the cluster beam deflector plates and Einzel lens focusing (midelement) electrode potential, the secondary ion current was maximized. The SIMS spectrum of choline chloride on a gold target has the virtually unique property of being insensitive to primary ion damage [13]. Under the conditions indicated, the beam of glycerol clusters (ions + neutral clusters) has an apparent diameter of about $2 \mathrm{~cm}$, as indicated by removal of oxidation from copper targets.

Samples are loaded onto targets that consist of 1.5mm-thick $1.6-\times 7.8-\mathrm{cm}$ copper plates. Four $1-\mathrm{cm}$-diam samples at positions indexed to the sample introduction-vacuum lock system of the microprobe can be loaded at one time. The secondary ion source has been designed specially for large samples, and the ion optics of the secondary ion source are adjusted by computer for each of 40,000 (i.e., $200 \times 200$ ) locations on the target that are bombarded by the primary ion beam to produce an image. Secondary ions extracted by the source are transmitted through the triple quadrupole tandem mass spectrometer and detected by a conversion dynode-continuous dynode electron multiplier as an analog current. Signal-to-noise (S/N) ratios reported for ion images are based on the amplified voltage that corresponds to a particular secondary ion intensity integrated over an arbitrary area of sample that contains the analyte. This value is then divided by the corresponding voltage integrated over an equal area of the tissue sample that does not contain the analyte. This type of $\mathrm{S} / \mathrm{N}$ measurement necessarily includes chemical noise. Pulse counting is not used with this system. Approximate values for absolute intensity are indicated for images (ions/pixel) with the assumption of a multiplier gain of $-10^{5} \mathrm{~V} / \mathrm{A}$.

Charge compensation is used for all samples [15]. As described previously, the method used employs continuous emission of a $5-10-\mathrm{eV}$ electron beam in a ribbon that traverses the sample in the absence of the secondary extraction field. The primary ion and extraction optics are alternately turned on and off to permit the electron beam to traverse the sample surface and electrons to be attracted to any point on the surface that has built up a charge. Unless otherwise specified, pertinent operational parameters of the system used for the results presented here are described in Table 1.

Secondary ion images were obtained by digital raster of the primary ion beam over the sample; that is, the primary ion spot was moved, then fixed on a location, and allowed to settle prior to data acquisition. The primary ion beam was pulsed on and off as part of the charge compensation scheme, and was turned off during changes in the primary ion deflector voltages for raster. Detector current was recorded for each point on the raster only during periods when the primary beam

Table 1. Instrumental parameters for the SIMS mass spectrometry microprobe

\begin{tabular}{ll}
\hline Parameter & Value \\
\hline \hline Primary ion species & $\mathrm{Cs}$ \\
Primary ion kinetic energy & $10.0 \mathrm{keV}$ \\
Primary ion current & $(0.25-1.8) \mathrm{nA}$ \\
Primary ion spot diameter & $100 \mu \mathrm{m}$ \\
Primary ion current density & $(2.5-18) \mu \mathrm{A} / \mathrm{cm}^{2}$ \\
Primary ion dose per image & $(0.2-1.5) \times 10^{12}$ ions $/ \mathrm{cm}^{2}$ \\
Primary ion dose per mass spectrum & $0.5-3.5 \times 10^{14}$ ions $/ \mathrm{cm}^{2}$ \\
Primary ion duty cycle & $(3.5 \mathrm{~ms}) /(4.5 \mathrm{~ms})$ \\
Raster size & $1 \mathrm{~cm} \times 1 \mathrm{~cm}$ \\
Image acquisition time & $180 \mathrm{~s}$ \\
Electron flood gun current & $200 \mu \mathrm{A}$ \\
Secondary ion energy & $10 \mathrm{eV}$ \\
Conversion dynode potential & $-6.0 \mathrm{kV}$ \\
Multiplier potential & $-2.0 \mathrm{kV}$ \\
Data acquisition rate & $50 \mathrm{kHz}$ \\
Data points per pixel & 128 \\
Pixel size & $50 \mu \mathrm{m}$ \\
\hline
\end{tabular}


was turned on, except for a slight correction for ion flight time through the mass analyzer. Image data (secondary ion and video) were processed for display by using the public domain program NIH Imnge [16].

For mass spectra, the primary ion beam was rastered over a small area of the sample; spectra were acquired at each point. As with imaging, the primary ion beam was pulsed for charge compensation and turned off while deflector voltages were adjusted. To generate areas on the sample subjected to a dose of primary ion beam in excess of the static SIMS limit, the primary $\mathrm{Cs}$ beam was rastered repeatedly over a small portion of the sample that contained some part of the spike of targeted analyte. The dose administered depended on the sample and was administered until periodically obtained mass spectra showed complete suppression of secondary ions characteristic of the analyte. This dose varied between $10^{14}$ and $10^{16}$ ions $/ \mathrm{cm}^{2}$.

\section{Sample Preparation}

Pork brain was purchased from a local supermarket, stored frozen, and thawed before sample preparation. Small portions $(\sim 10-20 \mu \mathrm{L})$ of the gelatinous mass were taken from the thawed mass and smeared onto a target. The thickness of such samples is estimated to be 50-100 $\mathrm{mm}$ (these are thick samples), and when dry, their appearance indicates a smooth surface. This target was then placed in vacuum and dried.

Acetylcholine chloride and choline chloride were obtained from Aldrich Chemical Co. (Milwaukee, WI) and used without further purification. Methylphenylpyridinium iodide was a gift from S. P. Markey. These compounds were dissolved in 0.1-M $\mathrm{HCl}$ to a concentration of approximately $0.1 \mathrm{M}$. Analytical samples were prepared by spotting a small volume (typically $2 \mu \mathrm{L}$ ) of analyte solution onto the tissue specimen mounted on the target. The sample was then dried under vacuum and introduced into the mass spectrometer. The approximate droplet size was 1-2-mm diameter. Our earlier results on differences in primary ion damage between neat analytes and analytes deposited in this manner suggest that the analyte penetrates the tissue [13]. Because of absorption by the tissue, the exact amount of material exposed at the surface is indeterminate.

\section{Results and Discussion}

\section{Choline Chloride on Tissue}

We have found that preparation of biological tissue is surprisingly difficult, and so we have confined our efforts to tissue that is most easily prepared. For the work presented here, brain tissue is used as the substrate, principally because porcine brain is readily available, is gelatinous, and can be smeared readily onto a target. Figure $1 \mathrm{a}$ and $\mathrm{b}$ shows contrast-enhanced video images of the tissue sample used to study the behavior of choline chloride. The methods of display reflect the two methods used throughout this paper to display secondary ion images. The data used for both images are identical, but the image presented in Figure $1 \mathrm{~b}$ shows more contrast. In Figure $1 \mathrm{c}$ and $d$, secondary ion images based on the intensity of $m / z 104$ are presented in both formats. No image enhancement is used for Figure $1 c$ and $d$ or any other ion images presented in this work. The white halo that surrounds the tissue sample is a common artifact that arises from chemical noise: secondary emission from a thin layer of organic compounds on metal targets is often greater than from the tissue and mainly arises from the various compounds that bleed from the sample as it dries. Tandem mass spectrometry can be used to remove such halos, provided they do not contain targeted analyte [13]. Use of tandem mass spectrometry necessarily requires a high dose of primary ions to the sample. Because tandem mass spectrometry substantially reduces chemical noise, which is one of the variables to be measured, tandem mass spectrometry was not used for the experiments reported here. The total dose of primary ions used to generate the image shown in Figure 1c is less than $10^{12} \mathrm{Cs}^{+} / \mathrm{cm}^{2}$, that is, well below the typical static SIMS limit of $10^{13}-10^{14}$ primary ions $/ \mathrm{cm}^{2}$.

Mass spectra of choline chloride spotted onto brain tissue are shown in Figure 2. Under static SIMS conditions, the intense peak at $m / z 104$ is characteristic of choline. After exposure to a dose of $10^{16} \mathrm{Cs}^{+} / \mathrm{cm}^{2}$, that is, about $100-1000 \times$ the typical static SIMS limit, any semblance of a characteristic choline spectrum vanishes. The prominent peak in the spectrum taken beyond the static SIMS limit is $m / z 133$, which is $\mathrm{Cs}^{+}$ implanted into the sample by the primary ion beam. For the same primary ion current, the intensity of the $m / z 133$ from the damaged region is only about $1 \%$ that of $m / z 104$ in the static SIMS spectrum of choline chloride. After exposure to about $30 \mathrm{~min}$ of the massive cluster beam, a spectrum taken under static SIMS conditions shows once again the intense $m / z 104$, which is the choline secondary ion. For comparison purposes, the absolute current observed for the $\mathrm{m} / \mathrm{z}$ 104 after exposure to the cluster beam is slightly less than half that detected from the undamaged sample.

The effect of primary ion damage to secondary ion images is disastrous. Figure $3 a$ shows the $m / z 104$ image of the tissue sample shown in Figure 1, that was taken under conditions of static SIMS and after a spike of choline chloride was deposited on it. Except for the spot in the center that corresponds to choline, Figures $1 \mathrm{c}$ and $3 \mathrm{a}$ are replicas. The image shown in Figure $3 \mathrm{~b}$ was obtained after the primary ion beam $\left(1.2 \mathrm{nA}, \mathrm{Cs}^{+}\right)$ had been aimed to strike only the right side of the spot and was rastered continuously over this right-side portion of the choline chloride spike for $4 \mathrm{~h}$. By damaging only part of the spike, the remainder of the spike could be used as an internal standard. It is clear that the primary ion beam obliterated the portion of choline 
a
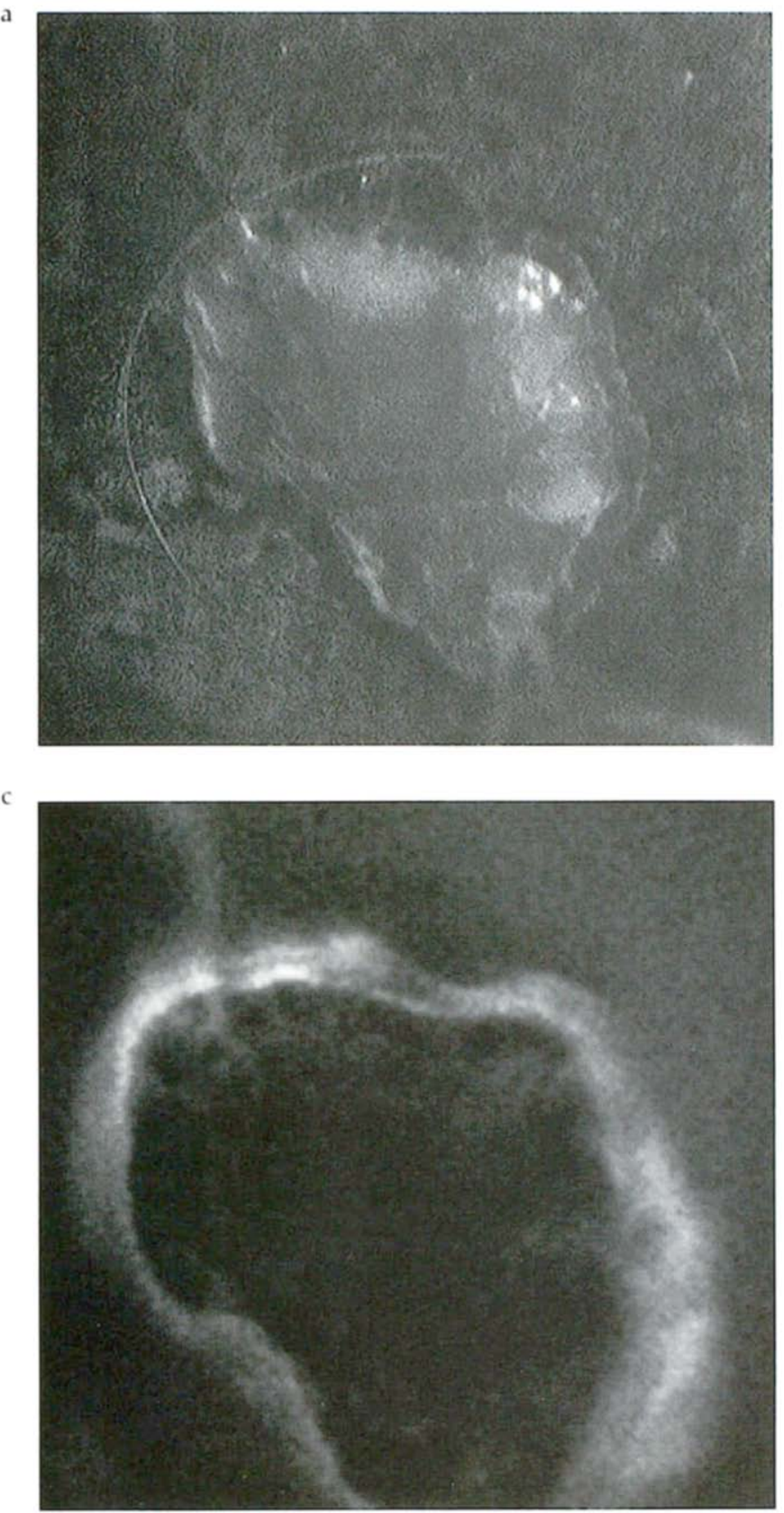

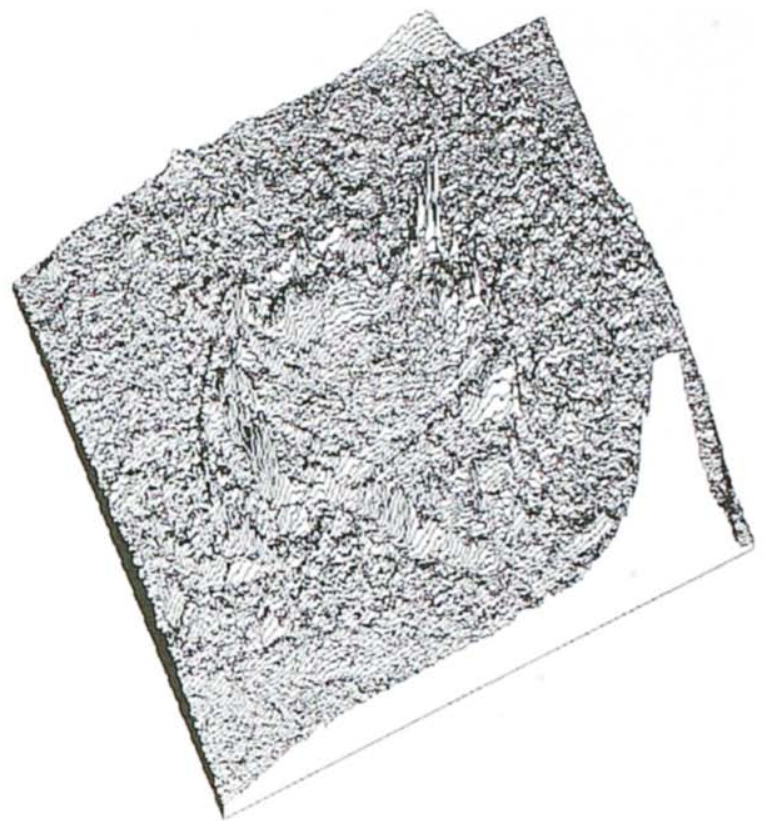

d

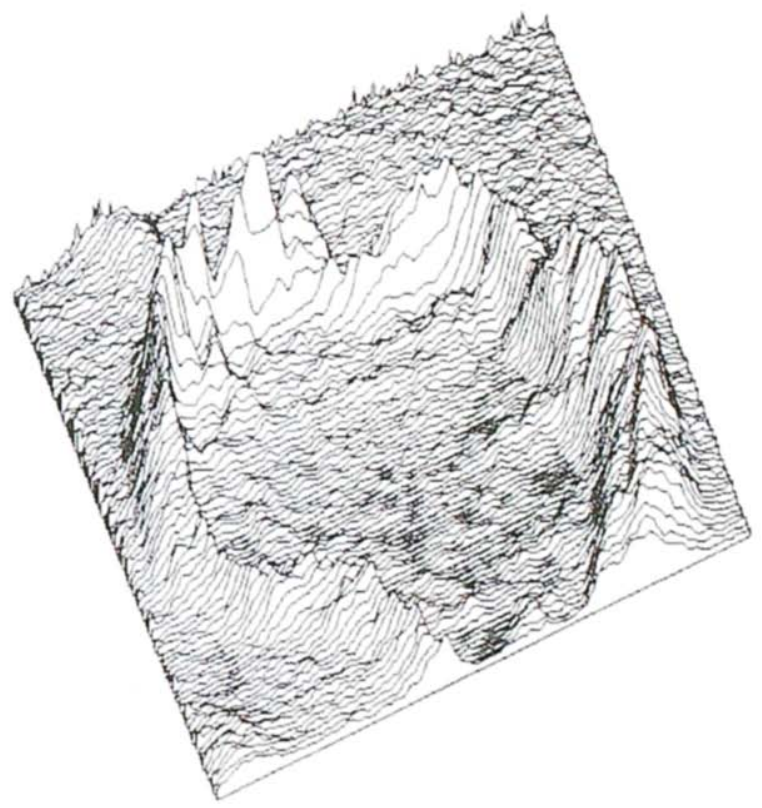

Figure 1. Reference images of pork brain sample prior to spotting a spike of choline solution. The data shown in the gray scale video image (a), are presented in the topographical format (b) used by the computer program NIH Image. (c) The gray scale image based on the secondary ion intensity of $m / z 104$, choline. For this image, the maximum intensity corresponds to 10,112 secondary ions/pixel and the minimum intensity corresponds to 0 secondary ions/pixel. Throughout this paper, the gray scale varies linearly with secondary ion intensity from minimum (black) to maximum (white). (d) The same data used for (c) are presented in topographical format.

chloride spike exposed to it-a result consistent with the absence of $m / z 104$ in the mass spectrum shown in Figure $2 \mathrm{~b}$. Although damaged, however, the choline is still present in the sample below the damaged region. This is evident from Figure $3 c$, which was obtained after $30 \mathrm{~min}$ of ablation by the massive cluster beam. This $m / z 104$ image was obtained by using the $\mathrm{Cs}^{+}$ primary ion beam under conditions of static SIMS and it clearly shows the return of choline secondary ion emission at a level that is $87 \%$ of its original value determined by static SIMS. The fact that images in Figure $3 a$ and $c$ indicate virtually the same choline distribution shows that massive cluster ablation does not disturb the lateral distribution of analyte. The 


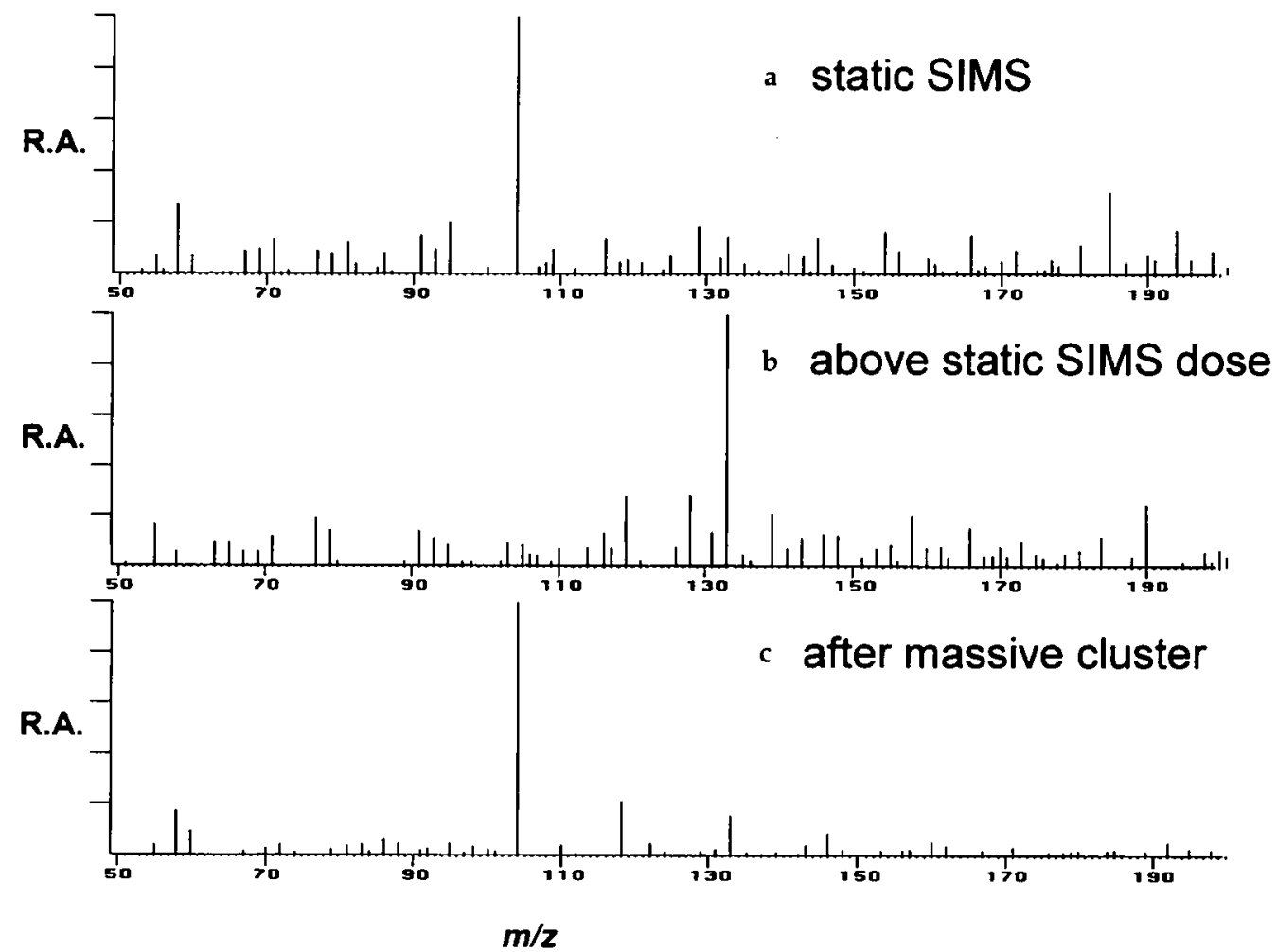

Figure 2. (a) The mass spectrum of choline spot placed on the tissue sample shown in Figure 1, under static SIMS conditions, shows an intense $m / z 104$. (b) This peak, which is taken from the portion of the sample exposed to a dose of $10^{1 \mathrm{~h}} \mathrm{Cs}^{+} / \mathrm{cm}^{2}$, is obscured. (c) The spectrum obtained from the damaged region after 30 -min exposure to the massive cluster beam.

demonstration that characteristic choline secondary ion emission could be suppressed by primary ion damage and then rejuvenated indicates that the spike penetrated the sample surface. As a neat sample, secondary ion emission from choline chloride ceases only when the sample has been depleted [13].

An important feature of Figure $3 c$ is the absence of the halo at the periphery of the sample; this halo is clearly evident in Figures $1 c$ and $3 a$ and $b$. As indicated in the foregoing text, this halo arises from material dissolved in the water contained in the tissue sample. Some of this solution flows from the sample as it dries, which leaves a film of organic residue at the periphery of the tissue. In general terms, the presence of such a halo implies that some motion of compounds on the tissue surface occurred during sample preparation and prior to drying. Evidence of material migration is the bette noir of mapping methods such as electron microscopy and inorganic SIMS [17]. Such evidence indicates that whatever distribution was determined was altered during sample preparation and does not necessarily reflect the distribution in vivo. A variety of sample preparation methods have been developed to avoid such migration [18]. These methods share the common features that they require expensive equipment (such as an ultracryomicrotome), are dif- ficult methods to master, and frequently involve the use of chemicals that are highly toxic.

Application of a massive cluster beam to the sample may remedy the effects of organic analyte migration in tissue. Inorganic ions such as $\mathrm{K}^{+}$and $\mathrm{Na}^{+}$can diffuse through membranes during the period required to cool living tissue to cryogenic temperatures [18]. Rapid transport of organic compounds may, however, be limited to surface migration. Consequently, removal of such material by the massive cluster beam may leave the remaining material as it was distributed in vivo. Admittedly, the spike of choline chloride appears to have penetrated the surface of the sample. Although the depth of penetration is not known, it is unlikely that it penetrated to a depth greater than a few micrometers. Lateral migration of this distance would be very close to the spatial resolution of most secondary ion microprobes and practically speaking, would be invisible.

\section{Acetylcholine Chloride and Methylphenylpyridinium lodide}

In previous work [6], we used choline chloride, acetylcholine chloride, and methylphenylpyridinium iodide $\left(\mathrm{MPP}^{+} \mathrm{I}^{-}\right)$as reference compounds because they have 


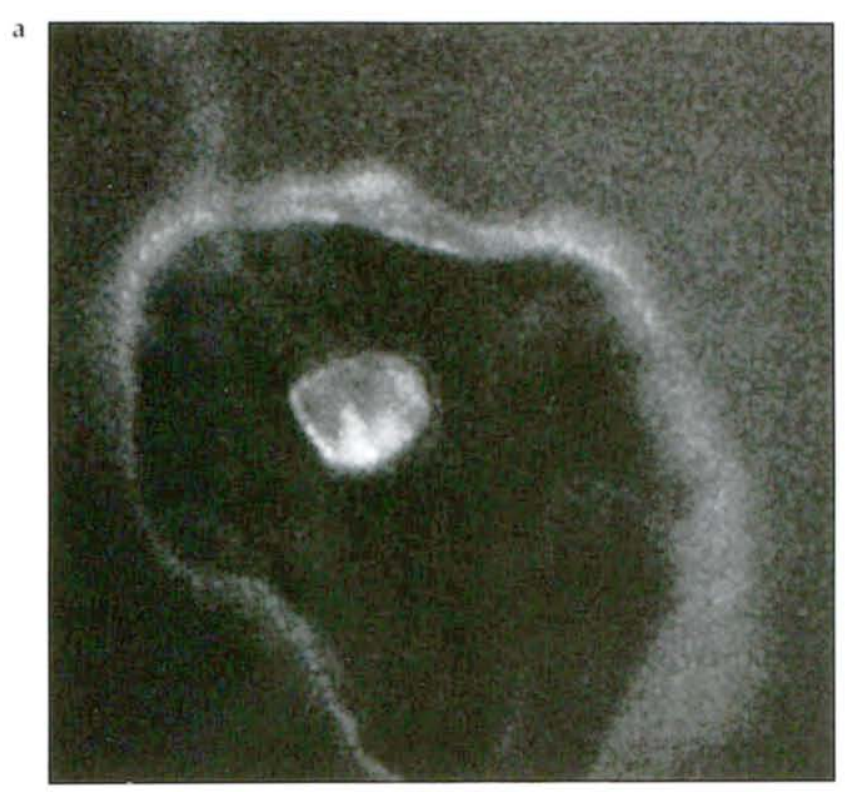

b
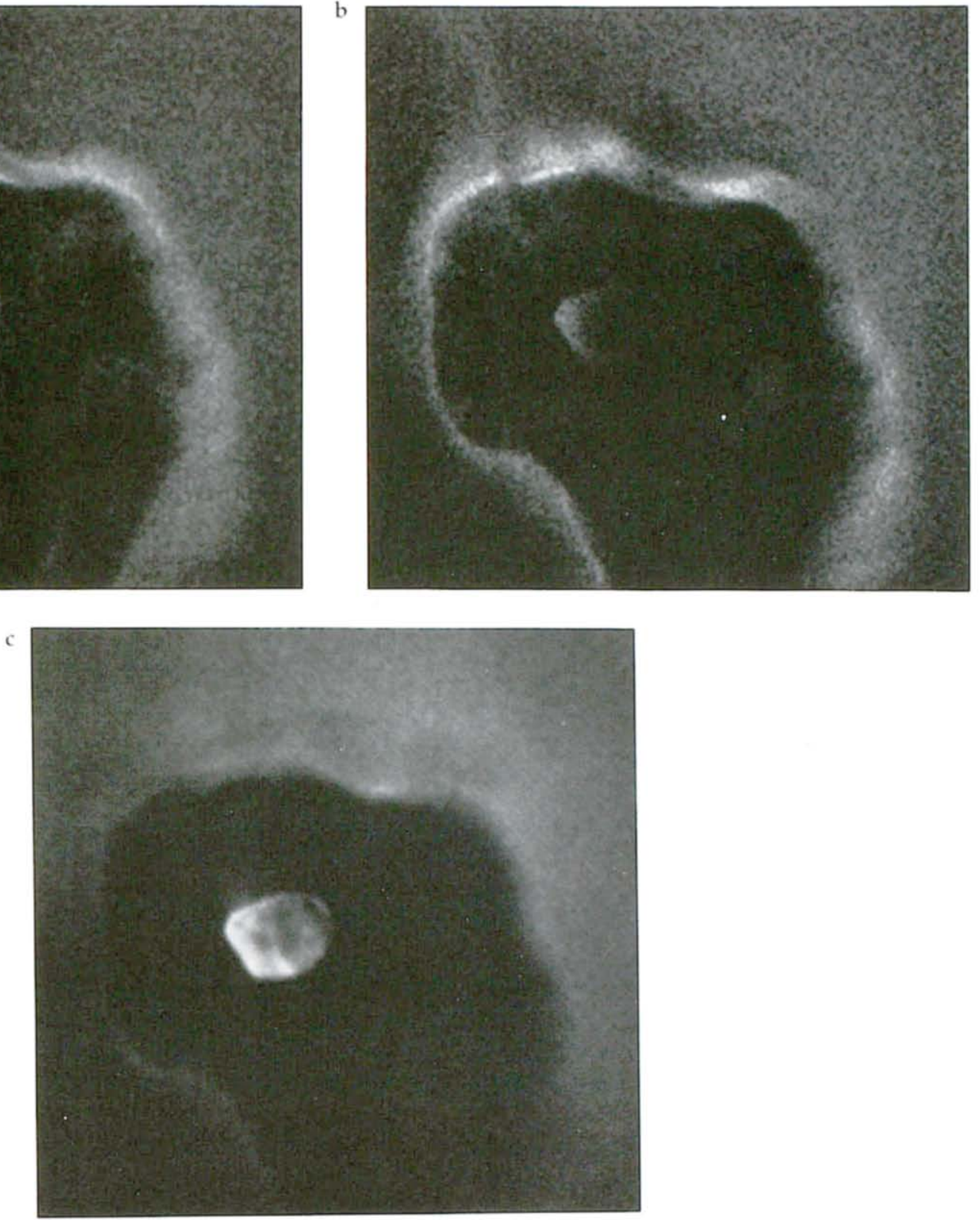

Figure 3. Images of $m / z 104$ (choline) (a) before and (b) after a portion of the sample in the vicinity of the sample spike was subjected to a dose of $10^{16} \mathrm{Cs}^{+} / \mathrm{cm}^{2}$. The dose required to produce static SIMS images is typically $10^{12} \mathrm{Cs}^{+} / \mathrm{cm}^{2}$. (c) After 30-min exposure to massive cluster beam, the image shows no evidence of damage by primary $\mathrm{Cs}^{+}$. The maximum intensities for (a) and (b) correspond to 7404 and 8689 secondary ions/pixel, respectively. Minimum intensity corresponds to 0 secondary ions/pixel.

high secondary ion yields in common and their SIMS spectra display a range of response to primary ion damage. The spectrum of choline chloride deposited on a gold target appears to be immune to the chemical effects of primary ion damage, for example, and secondary ion emission of $\mathrm{MPP}^{+}$from $\mathrm{MPP}^{+} \mathrm{I}^{-}$deposited on tissue decays more rapidly than that of choline and acetylcholine emission from choline chloride and acetylcholine chloride, respectively. Because their secondary ion emission characteristics with regard to damage by the primary ion beam are different than choline chloride, analyses of these compounds deposited as $2-\mu \mathrm{L}$ drops onto tissue was evaluated in the same manner as was done for choline chloride.

Figure 4 shows SIMS spectra obtained from a spot of acetylcholine chloride deposited onto a specimen of porcine brain tissue. The spectrum in Figure 4a was obtained by using the $\mathrm{Cs}^{+}$primary ion beam under conditions of static SIMS and is marked by characteristic peaks at $m / z 146$ and 87 , which is very similar to 
R.A.

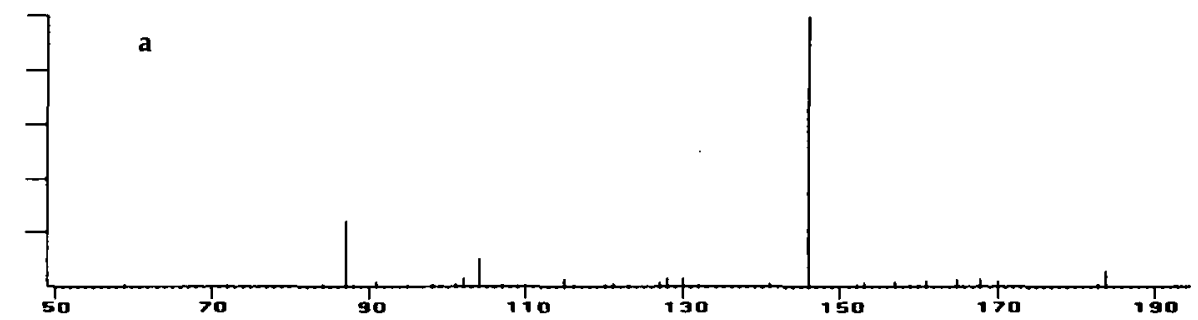

R.A.

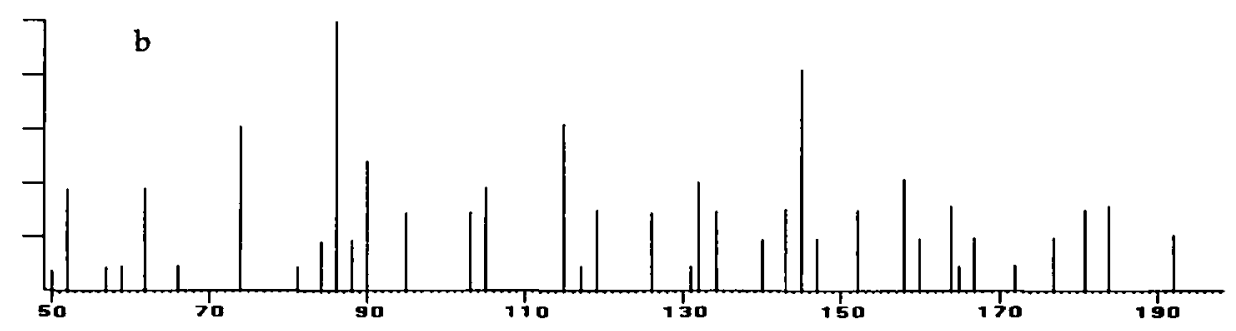

R.A.

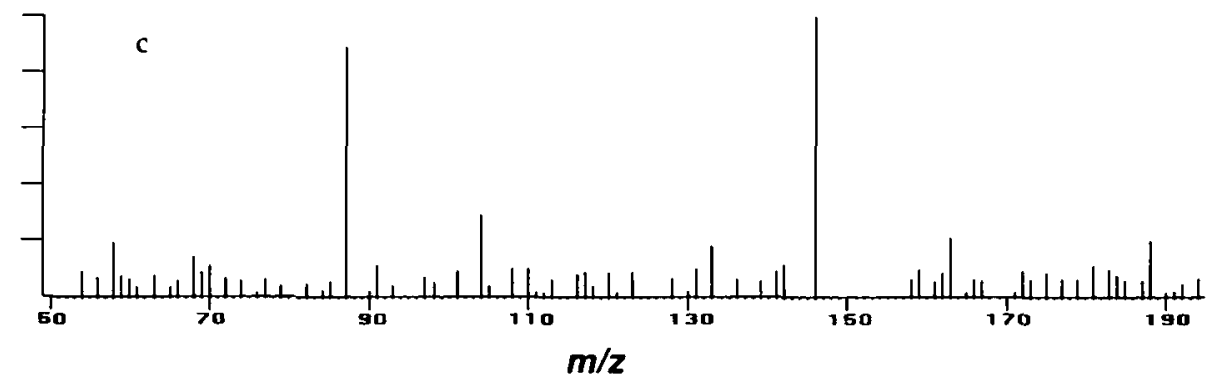

Figure 4. The static SIMS spectrum of acetylcholine spike on brain tissue. (a) The ion $m / 2146$ of acetylcholine and its major fragment, $m /=87$ are characteristic of acetycholine. (b) After exposure to a dose of $1.5 \times 10^{15} \mathrm{Cs}^{-} / \mathrm{cm}^{2}$, the mass spectrum is nonspecific. (c) After 30-min exposure to massive cluster beam, the characteristic $m / \approx 87$ and 146 return.

earlier reports [8]. The peak at $m / \approx 104$ arises from choline contamination, which is common in samples of acetylcholine. Beyond the static SIMS limit, however, no such prominent peaks are evident, as shown in Figure $4 \mathrm{~b}$, where any evidence of the presence of acetylcholine is obscured by chemical noise. After 30min ablation by the beam of massive clusters, however, the intensity of $m / z 146$ and 87 is restored, as shown in Figure 4c. The increase in the relative abundance of $m / \approx 87$ may be indicative of damage to the acetylcholine chloride spot by the massive clusters: Alternatively, some alteration of the tissue matrix itself by the cluster beam may cause this effect.

Figure 5 shows secondary $m / z 146$ ion images obtained from the sample that contains the acetylcholine chloride spike by using the topographical format of NIH Image [16]. The reference image in Figure 5 a was obtained under conditions well below the static SIMS limit. Figure $5 b$ clearly shows the removal of characteristic $m / z 146$ emission from the region of the spike subjected to a dose of primary $\mathrm{Cs}^{+}$above the static SIMS limit. After ablation by the massive cluster, secondary emission of $m / z 146$ is rejuvenated in the region of the spike as shown in Figure $5 c$, and surprisingly, appears more pronounced than the original image shown in Figure 5a. In fact, the absolute current of $m / z 146$ in Figure $5 c$ is only slightly greater than $35 \%$ that detected from the spike shown in Figure 5 a, but approximately $10 \times$ that from the damaged region shown in Figure 5b. The improvement in contrast shown in Figure $5 \mathrm{c}$ arises from suppression of chemical noise by the ablation of the massive cluster beam as well as an increase in characteristic secondary ion emission.

As previously noted, secondary ion emission of $\mathrm{MPP}^{\prime}$ is peculiarly sensitive to primary ion damage, particularly when MPP' $I$ is deposited on tissue. This is evident from the spectra shown in Figure 6. Under conditions of static SIMS, an intense $m /=170 \mathrm{MPP}^{\prime}$ is readily observed as shown in Figure $6 \mathrm{a}$. At a dose of $1.5 \times 10^{14} \mathrm{Cs} / \mathrm{cm}^{2}$, this emission is completely suppressed as shown in Figure 5b. Following 30-min ablation by a beam of massive clusters, secondary emission from the MPP ${ }^{\prime}$ is restored to $100 \%$; of its initial value.

The effect of such restoration is evident in the sequence shown in Figure 7 based on the intensity of $m / z$ 170. In Figure $7 \mathrm{a}$, emission from the $\mathrm{MPP}^{+} \mathrm{I}$ spike completely dwarfs emission from the tissue. In Figure $7 \mathrm{~b}$, emission from the region subjected to a large dose of $\mathrm{Cs}^{+}$is completely suppressed, whereas emission from the adjacent portion of the spike is unaffected. In Figure $7 c$, which was obtained after 30-min ablation, emission from the spike is returned, although it is somewhat less pronounced than the 

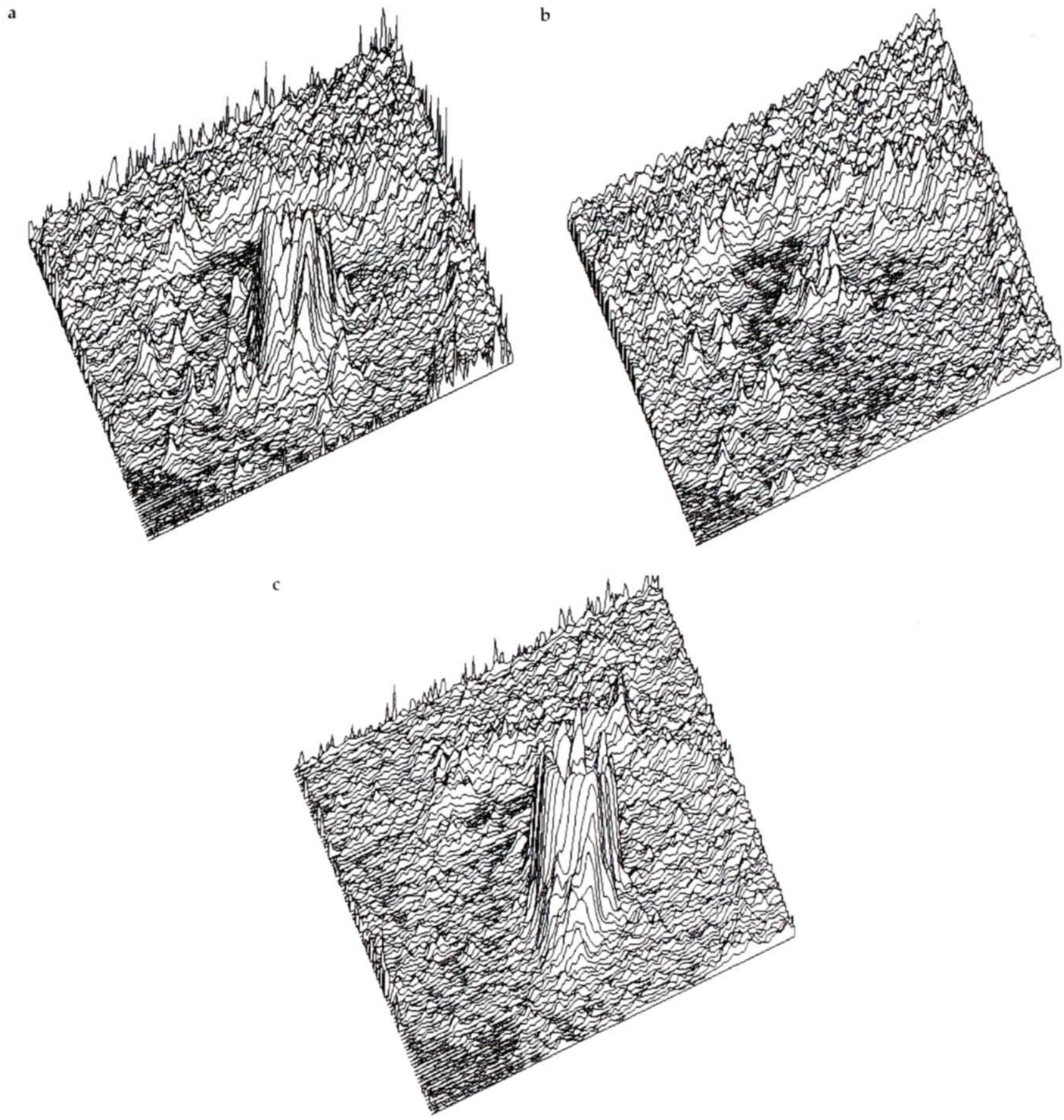

Figure 5. Secondary ion images of acetylcholine spot based on $m / z 146$ are displayed using the topographical format of NIH lmage (a) before and (b) after a portion of the spike had been exposed to a dose of $1.5 \times 10^{15} \mathrm{Cs}^{+} / \mathrm{cm}^{2}$. (c) The image subsequently obtained after 30-min exposure to the cluster beam. Maximum intensity, in secondary ions/pixel for these images is (a) 27,223, (b) 16,200, and (c) 8279. Minimum intensity corresponds to 0 secondary ions/pixel for all images.

initial image shown in Figure 7a. The spike at the top of the image shown in Figure $7 c$, and clearly from the metal substrate, is an artifact. It is worth noting briefly that background emission from the tissue in Figure 7c is less than that shown in Figure 7a, particularly when compared to that from the surrounding metal substrate. This kind of behavior depends upon a number of factors, which include isobaric interference and secondary ion yield from material, such as water and residual vapor from pump oil, which is adsorbed by the metal surface following ablation.

\section{Quantitative Evaluation of Performance}

The broad distributions in kinetic energy, charge state, and mass of the massive clusters used in these experiments do not permit detailed analysis of the usual parameters of penetration depth, range and stopping 

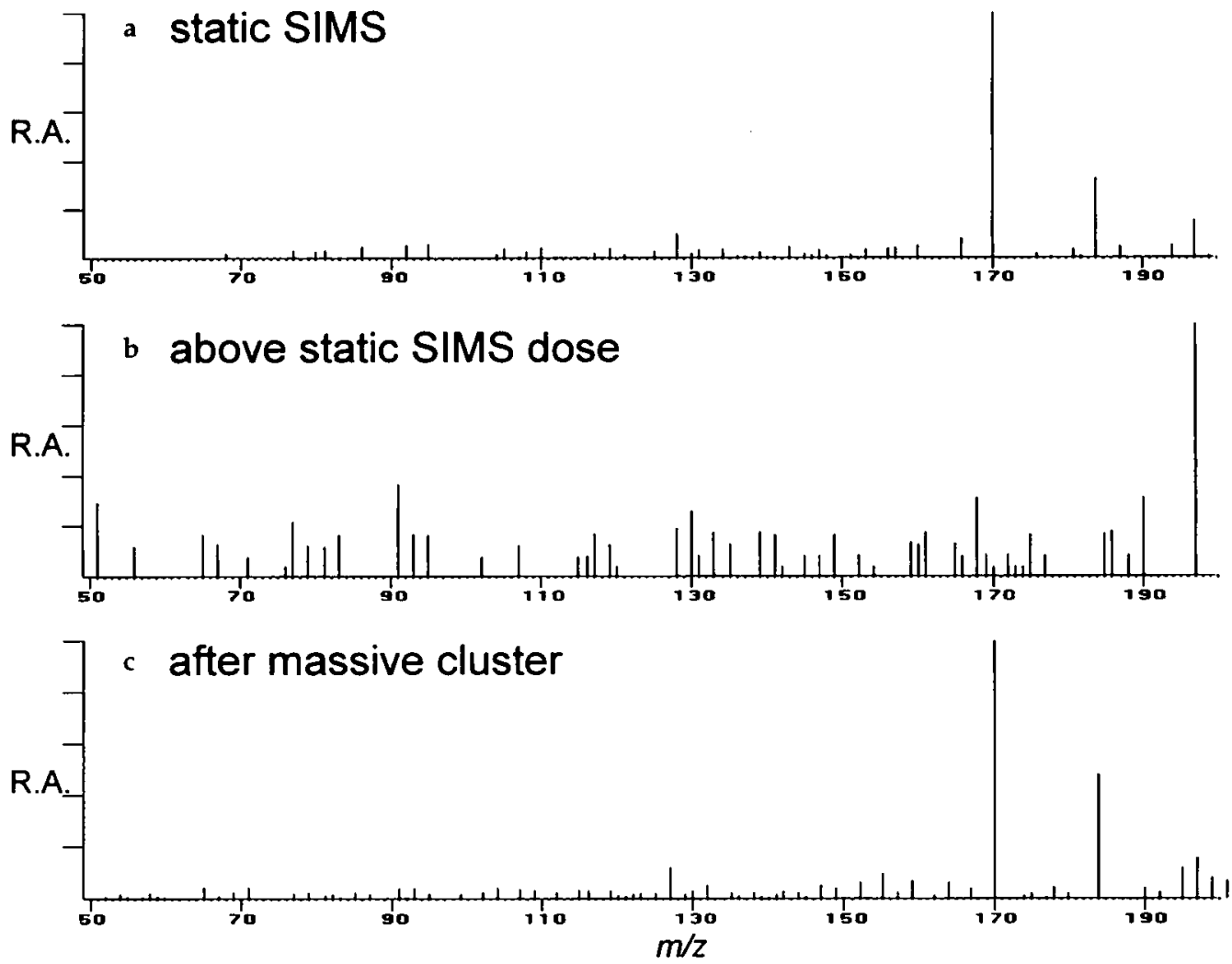

Figure 6. Mass spectra of methylphenylpyridinium (MPP' I ) obtained from spike on brain tissue (a) under static SIMS conditions, (b) after exposure to $1.5 \times 10^{1+} \mathrm{Cs}^{+} / \mathrm{cm}^{2}$, and (c) subsequent 30-min exposure to cluster beam shows results similar to those obtained with choline and acetylcholine. The intensity of MPP' is peculiarly sensitive to primary ion damage.

power, and so forth. Nonetheless, the cluster beam does rejuvenate secondary ion emission from samples subjected to a dose of primary ions demonstrably in excess of the static SIMS limit. As a measure of the effectiveness of massive cluster ablation as it relates to imaging tissue, the figure of merit is the signal-to-noise ratio. For the ion images presented here, the noise of interest is chemical noise. To evaluate this, we measured the sum of secondary ion intensity characteristic of analyte over the area of the spike and divided that number by the secondary ion intensity from an identical area on the sample, but away from the location of the spike. This operation is readily performed by using NIH Inage. Results from this analysis are shown in Table 2. Rejuvenation of emission appears to vary between about 10 and $80 \%$. With some optimization, it may be possible to improve these values. For example, the particular ablation time of 30 min was chosen based on experience with cleaning the surface of stearic acid. Until the flux and flux density of the cluster beam can be measured and adjusted, optimization experiments are premature. We are in the process of developing a dosimeter for this purpose.

Even if, after rejuvenation, secondary ion current is only $10 \%$ of its value under conditions of static SIMS, repetitive sampling and ablation permit signal averaging. The potential of the massive cluster beam for organic tissue analysis appears to be in enabling the use of tandem mass spectrometry and summing of images. In previous work, we reported the capability to perform both operations under static SIMS conditions, although with samples where the analyte was fairly concentrated. Repetitive cycling of the cluster beam is a prerequisite for extension of these methods to trace analysis. Development of such a capability is currently underway.

\section{Conclusions}

Primary ion damage is an unavoidable consequence of organic analysis and imaging by SIMS. Traditionally, such damage has included sputtering, primary ion implantation, and chemical alteration of the sample, as evidenced by changes in the spectrum or image. All these effects apparently are operative regardless of whether the sample is subjected to a beam of primary $\mathrm{Cs}^{+}$or a beam of massive clusters. The major difference between the damage to the sample caused by monatomic primary ion beams and massive cluster beams is the ratio of sputtering to implantation and 


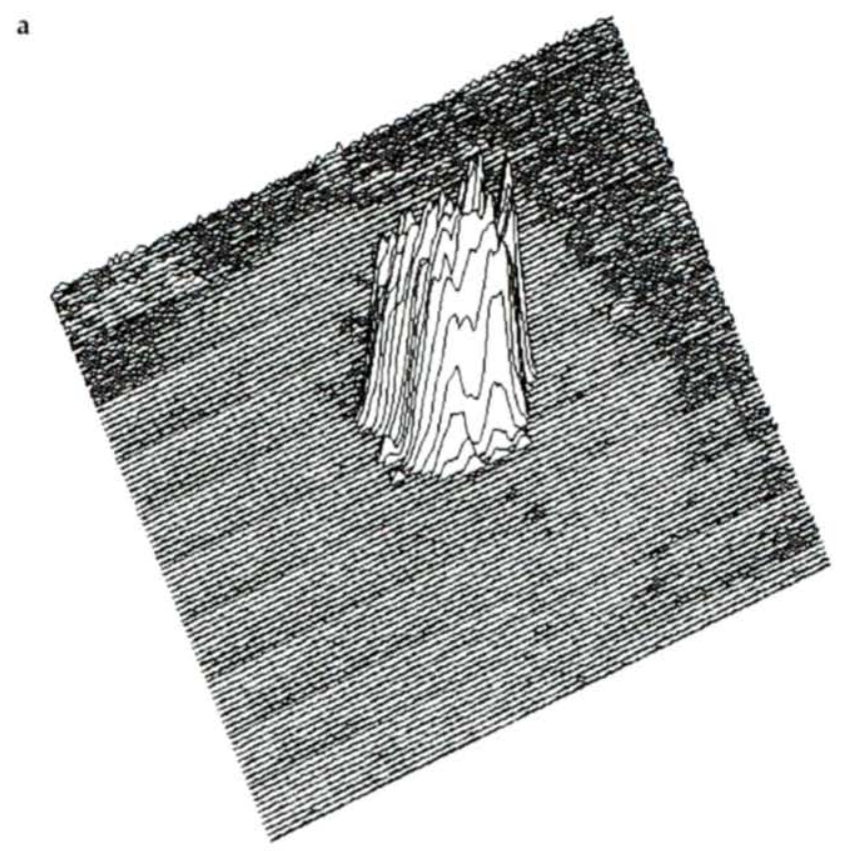

b
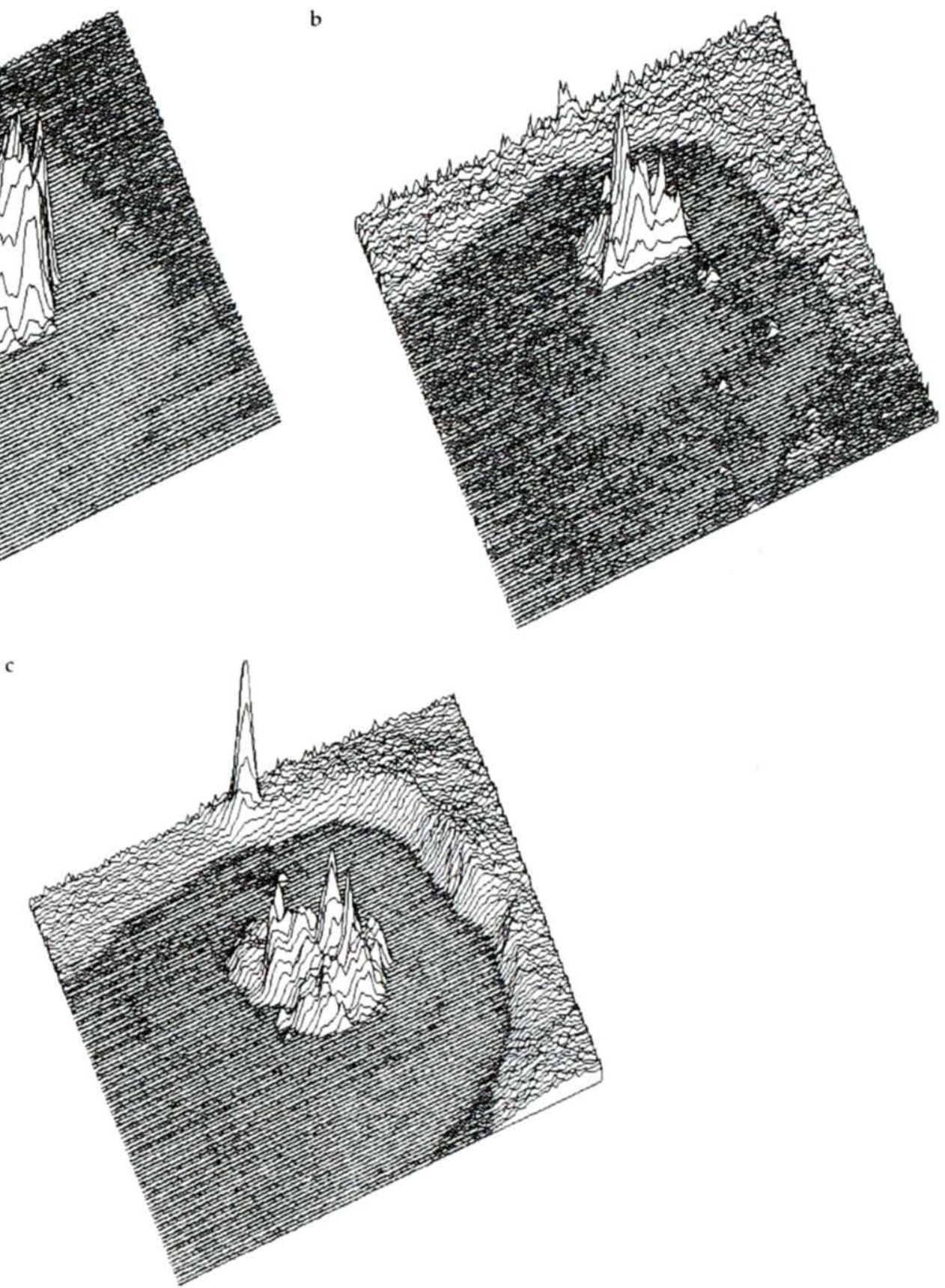

Figure 7. Images of $\mathrm{MPP}^{+}$spike on brain tissue based on the intensity of $m /=170$ (a) under static SIMS conditions, (b) after exposure to $1.5 \times 10^{14} \mathrm{Cs}^{+} / \mathrm{cm}^{2}$ and (c) after 30-min exposure to cluster beam are consistent with results obtained with choline and acetylcholine. The spike at the top of the image is from chemical noise that arises from the metal target. The maximum secondary ion intensity/pixel for these images is (a) 41,626, (b) 12,236, and (c) 41,968. Minimum intensity corresponds to 0 secondary ions/pixel for all images.

chemical alteration. Removal of the halo observed in Figure $1 \mathrm{c}$ indicates that the massive cluster gun rejuvenates secondary ion emission by ablation of damaged molecules; that is, the ratio of sputtering to other forms of damage is relatively high for impact of massive clusters.

Although any number of mechanisms could be invoked to rationalize the behavior of secondary ion emission after cluster ablation, the significance of mas- sive cluster impact lies in the practical. Specifically, application of massive cluster impact permits spatially resolved chemical analysis of biological tissue beyond the static SIMS limit.

\section{Acknowledgment}

We are grateful to S. P. Markey and J. T. Brenna for their copoperation and insight in development of this instrumentation 
Table 2. Signal-to-noise and primary ion dose

\begin{tabular}{lccc}
\hline Secondaryion & Static SIMS & Damaged & $\begin{array}{c}\text { After massive } \\
\text { cluster ablation }\end{array}$ \\
\hline \hline Choline & 174 & 5 & 153 \\
Acetylcholine & 1295 & 15 & 154 \\
$\begin{array}{l}\text { Methylphenyl- } \\
\text { pyridinium }\end{array}$ & 2438 & 1 & 630 \\
$\left(\mathrm{MPP}^{+}\right)$ & & & \\
\hline
\end{tabular}

and for their helpful discussions. The ion microprobe is on loan to ORNL from the Biomedical and Instrumentation Engineering Program, National Center of Research Resources, National Institutes of Health. Development of fundamental properties of massive cluster impact was sponsored by the U.S. Department of Energy, Office of Basic Energy Sciences, under contract DE-AC0584OR21400 with Martin Marietta Energy Systems. Application of the massive cluster impact source to organic ion imaging was sponsored by the National Institutes of Health under Grant RO1-GM41617. This research was supported in part by N. N. Dookeran's appointment to the Oak Ridge National Laboratory Postdoctoral Research Program administered jointly by Oak Ridge Institute for Science and Education and Oak Ridge National Laboratory.

\section{References}

1. Benninghoven, A.; Sichtermann, W. K. Annl. Chem. 1978, 50 , 1180.
2. Busch, K.; Glish, G. L.; McLuckey, S. A. Mass Spectrometry / Mass Spectrometry; VCH Publishers: Deerfield, FL, 1988.

3. Cole, R. B.; Guanat, C.; Haas, J. J.; Linton, R. W. Anal. Chem. 1987, 59, 1930.

4. Barber, M.; Bordoli, R. S.; Elliott, G. J.; Sedgwick, R. D.; Tyler, A. N. Anal. Chem. 1982, 54,645A.

5. Wong, S. S.; Röllgen, F. W.; Manz, I.; Przybylski, M. Biomed. Mass Spectrom. 1985, 12, 43.

6. Kriger, M. S.; Cook, K. D.; Short, R. T.; Todd, P. J. Anal. Chem. 1992, 64, 3052.

7. Ligon, W. V.; Dorn, S. B. Int. J. Mass Spectrom. Ion Processes 1984, 57, 75.

8. Gillen, G.; Simons, D. S.; Williams, P. Anal. Chem. 1990, 62, 2122.

9. Cornett, D. S.; Lee, T. D.; Mahoney, J. F. Rapid Commun. Mass Spectrom. in press.

10. Mahoney, J. F.; Perel, J.; Ruatta, S. A.; Martino, P. A.; Husain, S.; Lee, T. D. Rapid Commun. Mass Spectrom. 1991, 5, 441.

11. Mahoney, J. F.; Perel, J.; Lee, T. D.; Martino, P. A.; Williams, P. J. Am. Soc. Mnss Spectrom. 1992, 3, 311.

12. Ziegler, J. F.; Biersack, J. P.; Littmark, U. The Stopping and Range of lons in Solids; Pergamon: New York, 1985.

13. Todd, P. J.; McMahon, J. M.; Short, R. T. lnt. J. Mass Spectrom. lon Processes 1995, 143, 131.

14. Grimm, C. C.; Short, R. T.; Todd, P. J. I. Am. Soc. Mass Spectrom. 1991, 2, 362.

15. Short, R. T.; McMahon, J. M.; Holland, W. M.; Todd, P. J. J. Am. Soc. Mass Spectrom. 1994, 5, 37.

16. Rasband, W. NIH lmage 1.43, 1992, Internet or BitNet: wayne@helix.nih.gov.

17. Brenna, J. T.; Morrison, G. H. Anal. Chem. 1986, 58, 1675.

18. Hayat, M. A. Principles and Teclniques of Electron Microscopy Biologicnl Applicntions, 3rd ed.; CRC Press: Boca Raton, FL, 1989. 